\title{
Acceleration Statistics of Inertial Particles from High Resolution DNS Turbulence
}

Federico Toschi ${ }^{1}$, Jeremie Bec ${ }^{2}$, Luca Biferale ${ }^{3}$, Guido Boffetta ${ }^{4}$, Antonio Celani $^{5}$, Massimo Cencini ${ }^{6}$, Alessandra S. Lanotte ${ }^{7}$, and Stefano Musacchio ${ }^{8}$

1 CNR-IAC, Viale del Policlinico 137, I-00161 Roma, Italy and INFN, Sezione di Ferrara, via G. Saragat 1, I-44100, Ferrara, Italy. toschi@iac.cnr.it

2 CNRS Observatoire de la Côte d'Azur, B.P. 4229, 06304 Nice Cedex 4, France

3 Dept. of Physics and INFN, University of Rome "Tor Vergata", Via della Ricerca Scientifica 1, 00133 Roma, Italy

4 Dept. of Physics and INFN, University of Torino, Via Pietro Giuria 1, 10125, Torino, Italy

${ }^{5}$ CNRS, INLN, 1361 Route des Lucioles, F-06560 Valbonne, France

6 SMC-INFM c/o Dept. of Physics University of Rome "La Sapienza", Piazz.le A. Moro, 2, I-00185 Roma, Italy, and CNR-ISC via dei Taurini, 19 I-00185 Roma, Italy

7 CNR-ISAC and INFN, Sezione di Lecce, Str. Prov. Lecce-Monteroni km.1200, I-73100 Lecce, Italy

8 Dept. of Physics and INFN, University of Torino, Via Pietro Giuria 1, 10125, Torino, Italy

\begin{abstract}
We present results from recent direct numerical simulations of heavy particle transport in homogeneous, isotropic, fully developed turbulence, with grid resolution up to $512^{3}$ and $R_{\lambda} \approx 185$. By following the trajectories of millions of particles with different Stokes numbers, $S t \in[0.16: 3.5]$, we are able to characterize in full detail the statistics of particle acceleration. We focus on the probability density function of the normalised acceleration $a / a_{\mathrm{rms}}$ and on the behaviour of their rootmean-squared acceleration $a_{\mathrm{rms}}$ as a function of both $S t$ and $R_{\lambda}$. We explain our findings in terms of two concurrent mechanisms: particle clustering, very effective for small $S t$, and filtering induced by finite particle response time, taking over at larger $S t$.
\end{abstract}

Keywords: Lagrangian turbulence, heavy particles, Stokes particles, acceleration statistics

\section{Introduction}

Small impurities, as for example dust, droplets or bubbles, suspended in an incompressible flow are finite-size particles whose density, in general, differs from that of the underlying advecting fluid. Hence such particles cannot be modeled as point-like tracers. The description of their motion must account for

Y. Kaneda (ed.), IUTAM Symposium on Computational Physics and New Perspectives in Turbulence, 73-78.

(c) 2008 Springer. 
inertia whence the name inertial particles. Ultimately such inertial particles concentrate onto nonuniform sets that evolve together with the fluid motion and display strong spatial inhomogeneity, often called preferential concentration (see [1] in this same volume). The role of inertia is also reflected into a slow response of these particles to changes in the fluid velocity. The combined effects of non-uniform sampling of the physical space (preferential concentration) and of the finite response time of the particles explain the observed behaviour of particle acceleration.

\section{Heavy Particle Dynamics and Numerical Simulations}

The equations of motion of a small, rigid, spherical particle immersed in an incompressible flow have been derived from first principles in [2]. For particles much heavier than the surrounding fluid, these equations take the form

$$
\frac{d \boldsymbol{X}}{d t}=\boldsymbol{V}(t), \quad \frac{d \boldsymbol{V}}{d t}=-\frac{\boldsymbol{V}(t)-\boldsymbol{u}(\boldsymbol{X}(t), t)}{\tau_{s}} .
$$

Here, $\boldsymbol{X}(t)$ denotes the particle trajectory, $\boldsymbol{V}(t)$ its velocity, $\boldsymbol{u}(\boldsymbol{x}, t)$ is the fluid velocity. The Stokes response time is $\tau_{s}=2 \rho_{p} a^{2} /\left(9 \rho_{f} \nu\right)$ where $a$ is the particle radius $\rho_{p}$ and $\rho_{f}$ are the particle and fluid density, respectively, and $\nu$ is the fluid kinematics viscosity. The Stokes number is defined as $S t=$ $\tau_{s} / \tau_{\eta}\left(\tau_{\eta}=(\nu / \epsilon)^{1 / 2}\right.$ being the Kolmogorov timescale and $\epsilon$ the average rate of energy injection). Equation (1) holds for very dilute suspensions, where particle-particle interactions (collisions) and hydrodynamic coupling can be neglected. The fluid evolves according to the incompressible Navier-Stokes equations:

$$
D_{t} \boldsymbol{u}=\left(\partial_{t} \boldsymbol{u}+\boldsymbol{u} \cdot \boldsymbol{\nabla} \boldsymbol{u}\right)=-\frac{1}{\rho_{f}} \nabla p+\nu \Delta \boldsymbol{u}+\boldsymbol{f}, \quad \boldsymbol{\nabla} \cdot \boldsymbol{u}=0,
$$

where $p$ is the pressure field and $\boldsymbol{f}$ is the external energy source, $\langle\boldsymbol{f} \cdot \boldsymbol{u}\rangle=\epsilon$.

The Navier-Stokes equations are solved on a (three-periodic) cubic grid of size $N^{3}$. Forcing is realized by keeping constant the spectral content of the two smallest wavenumber shells [3]. Viscosity is chosen by requiring a Kolmogorov lengthscale $\eta \approx \Delta x$ ( $\Delta x$ being the grid spacing): this choice ensures that the small-scale velocity dynamics is well resolved. We used a fully dealiased pseudospectral algorithm with $2 n d$ order Adam-Bashforth time-stepping.

Point particles are seeded homogeneously and with velocities equal to the local fluid velocity of a thermalized configuration. After a transient of about half large scale eddy turn over time the Lagrangian dynamics becomes stationary, and measurements are performed. We follow 15 sets of inertial particles with Stokes numbers in the range $[0.16: 3.5]$ and for each set, we store at high-frequency the position, the velocity of the particles, the velocity of the carrier fluid. Fluid tracers $(S t=0)$, evolving as $d \boldsymbol{x}(t) / d t=\boldsymbol{u}(\boldsymbol{x}(t), t)$, are also followed for comparison. A summary of relevant physical parameters is given in Table 1. 
Table 1. Parameters of our DNS. Microscale Reynolds number $R_{\lambda}$, root-meansquare velocity $u_{\mathrm{rms}}$, energy dissipation $\varepsilon$, viscosity $\nu$, Kolmogorov lengthscale $\eta=\left(\nu^{3} / \varepsilon\right)^{1 / 4}$, integral scale $L$, large-eddy Eulerian turnover time $T_{E}=L / u_{\mathrm{rms}}$, Kolmogorov timescale $\tau_{\eta}$, total integration time $T_{t o t}$, duration of the transient regime $T_{t r}$, grid spacing $\Delta x$, resolution $N^{3}$, number of trajectories of inertial particles for each Stokes $N_{t}$ dumped at frequency $\tau_{\eta} / 10$, number of particles $N_{p}$ per Stokes dumped at frequency $10 \tau_{\eta}$, total number of advected particles $N_{t o t}$. Typical errors on all statistically fluctuating quantities are of the order of $10 \%$.

\begin{tabular}{ccccccccccccccc}
\hline$R_{\lambda}$ & $u_{\mathrm{rms}}$ & $\varepsilon$ & $\nu$ & $\eta$ & $L$ & $T_{E}$ & $\tau_{\eta}$ & $T_{\text {tot }}$ & $T_{\text {tr }}$ & $\Delta x$ & $N^{3}$ & $N_{t}$ & $N_{p}$ & $N_{\text {tot }}$ \\
\hline 185 & 1.4 & 0.94 & 0.00205 & 0.010 & $\pi$ & 2.2 & 0.047 & 14 & 4 & 0.012 & $512^{3}$ & $5 \cdot 10^{5}$ & $7.5 \cdot 10^{6}$ & $12 \cdot 10^{7}$ \\
105 & 1.4 & 0.93 & 0.00502 & 0.020 & $\pi$ & 2.2 & 0.073 & 20 & 4 & 0.024 & $256^{3}$ & $2.5 \cdot 10^{5}$ & $2 \cdot 10^{6}$ & $32 \cdot 10^{6}$ \\
65 & 1.4 & 0.85 & 0.01 & 0.034 & $\pi$ & 2.2 & 0.110 & 29 & 6 & 0.048 & $128^{3}$ & $3.1 \cdot 10^{4}$ & $2.5 \cdot 10^{5}$ & $4 \cdot 10^{6}$ \\
\hline
\end{tabular}

\section{Results}

Here we briefly recall recent results on the statistical properties of acceleration [7]. The main signature of inertia on acceleration can be appreciated from Fig. 1 (left), where we show the normalized root mean squared acceleration, $a_{r m s}=\sqrt{\left\langle|\boldsymbol{a}|^{2}\right\rangle / 3}$. As the Stokes number increases a sharp decrease of $a_{r m s}$ can be observed (particularly for small $S t$ values). At changing the Reynolds number, we find an overall dependence very similar to that observed for tracers [4]. Let us now understand how this fall off is generated. In Fig. 1 (right) we plot the root mean square fluid acceleration conditioned on the particle positions, $D_{t} \boldsymbol{u}(\boldsymbol{X}(t), t)$. For small Stokes numbers such quantities collapse onto the curve of the particle acceleration. This tells us that the fast fall off of acceleration for small $S t$ can be fully explained in terms of the "preferential concentration" of particles: particles do not sample uniformly the space. Indeed they tend to be ejected from vortex filaments, which are characterized by high acceleration [5].

However, at larger Stokes number the conditional acceleration tends to recover the value it has in the case of tracers, meaning that as $S t$ increases in the range here explored-, an homogeneous sampling of the small scale flow structures is recovered (see also [1]). Therefore preferential concentration is not the only mechanisms influencing particle acceleration statistics. For this we note that the particle equation of motion (1) can be formally solved as $\boldsymbol{V}(t)=\int_{-\infty}^{t} e^{-(t-s) / \tau_{s}} \boldsymbol{u}(\boldsymbol{X}(s), s) d s$ which tells us that inertia plays a role similar to that of an exponential low-pass filter for the fluid velocity. Of course this is not exactly true because particle trajectories are different from those of tracers. We can define a filtered fluid velocity along tracers trajectories, i.e. $\boldsymbol{u}^{F}(t)=\int_{-\infty}^{t} e^{-(t-s) / \tau_{s}} \boldsymbol{u}(\boldsymbol{x}(s), s) d s$ and compute the acceleration $\boldsymbol{a}^{F}=\frac{d}{d t} \boldsymbol{u}^{F}$. By averaging $\left|\boldsymbol{a}^{F}\right|^{2}$ along the tracer trajectories we eliminate any effect of preferential concentration and focus on the effect of filtering. As one can see from 1 (right), for large $S t$, one has a fairly good agreement between the $a_{r m s}$ of the inertial particles and the root mean squared acceleration computed on 

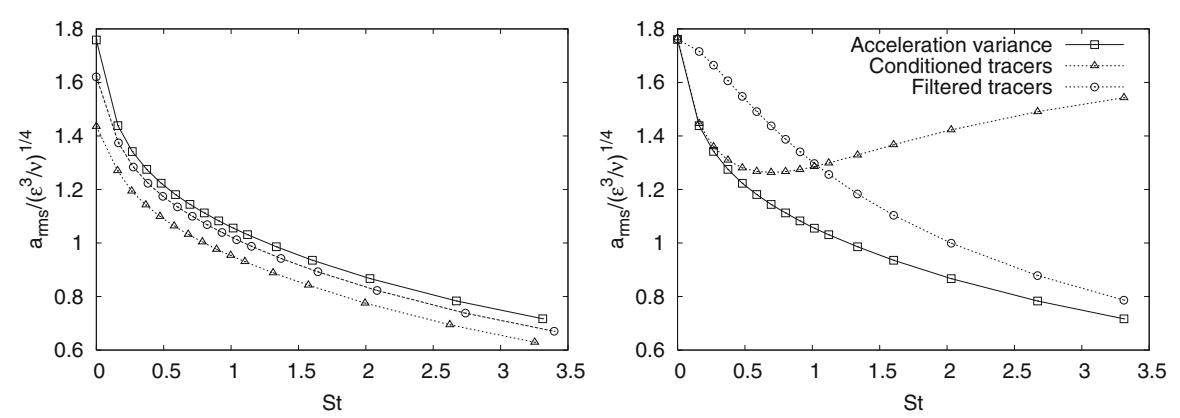

Fig. 1. (left) Normalised acceleration variance $a_{\mathrm{rms}} /\left(\epsilon^{3} / \nu\right)^{1 / 4}$ vs. $S t$ at varying the Reynolds number: $R_{\lambda}=185(\square) ; R_{\lambda}=105(\circ) ; R_{\lambda}=65(\triangle)$. (right) Acceleration variance, $a_{\mathrm{rms}}(\square)$, vs. $S t$, compared with the fluid acceleration at particle position, $\left\langle\left(D \boldsymbol{u} / D t(\boldsymbol{X}(t), t)^{2}\right\rangle^{1 / 2}(\triangle)\right.$ and with the root mean square acceleration of filtered tracer trajectories, $a_{\mathrm{rms}}^{F}(\circ)$. Data in this figure refer to $R_{\lambda}=185$.
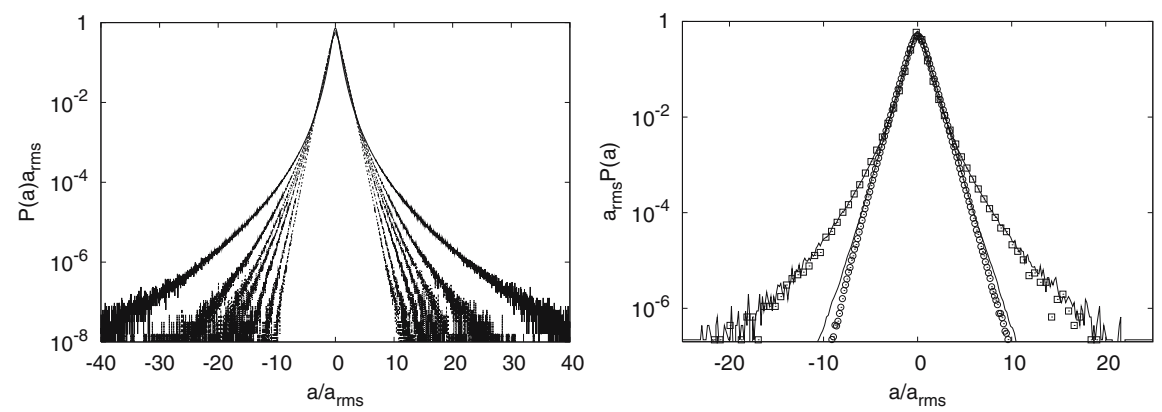

Fig. 2. (left panel) Acceleration probability distribution function for a subset of Stokes values $(S t=0,0.16,0.37,0.58,1.01,2.03,3.31$ from top to bottom, respectively) and for the case $R_{\lambda}=185$. (right panel) The two more external curves correspond to the acceleration pdf for $S t=0.16(\square)$ and the pdf of the fluid tracers acceleration measured at the same position of the inertial particles, $\frac{D \boldsymbol{u}}{D t}$ (solid line). The two inner curves are the acceleration pdf for the highest Stokes number, $S t=3.31$, (o) and the pdf of the filtered fluid acceleration (solid line). All curves are normalised to unit variance.

filtered (over a time window $\tau$ ) tracer trajectory. This shows that, for those values of $S t$, the main role of inertia is to act as a filter for the high-frequency (intense) fluctuations of the fluid acceleration.

In Fig. 2 we show the acceleration probability density functions for some particular $S t$ values. It is evident that the effect of increasing $S t$ does produce a tendency for the acceleration pdfs to have lower and lower tail. Thanks to the previous observations, one is tempted to interpret this steepening of the pdf's tails as due to the combined effect of preferential concentration and filtering. Figure 2 demonstrates that this is indeed correct. Here we compare the normalised pdf of the particle acceleration with that of the fluid tracers 
conditioned to be at particle positions (for a small $S t$ value) and the filtered tracer acceleration for a large $S t$ value. As one can see the agreement between the entire functional form of the acceleration pdf is striking and confirms the relevance of preferential concentration or filtering in the two opposite limiting case of, respectively, small or large $S t$ value. Besides the phenomenological picture for the behaviour of the acceleration, one may be interested in assessing how much our simplified model, represented by (1), do indeed describe physical reality. For the sake of this comparison we refer to recent experiments reported in [6]. A first observation is that in experiments it is impossible to deal with an exactly monodispersed phase, i.e. the radii of the particles (droplets) is distributed around some mean value. As a consequence this implies that the particles will not have the same Stokes number but a distribution peaked around a mean value. However experiments [6] were so accurate that the variance of the particle size distribution was precisely controlled and the particles could almost be regarded as monodisperse. Indeed we do find an excellent agreement between experimental and numerical pdf for the acceleration (see Fig. 3) at comparable Stokes numbers.

As a side note, the huge database that we have collected, in principle, allows us to make predictions also for polydispersed solution. If the solution is polydispersed one would measured, e.g. for the acceleration, the convolution of the acceleration measured for a give Stokes with the relative probability to find such a Stokes particle. Analysis in this direction has beet attempted in $[8]$.

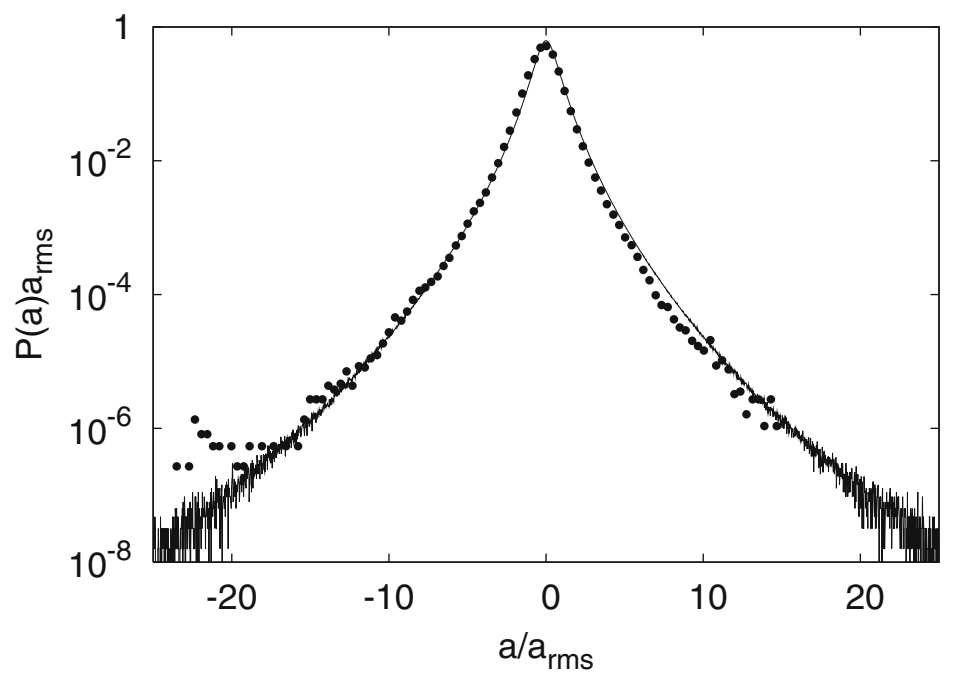

Fig. 3. Comparison between experimental acceleration pdf $(\bullet)$ at $\langle S t\rangle=0.09 \pm 0.03$ from [6] and numerical data (solid line) at $S t=0.16$ from $[7,8]$. 


\section{Conclusions}

We presented results on the statistical properties of acceleration for inertial particles in turbulent flows. We have shown that for small $S t$ values the predominant effect can be associated to a tiny change in the space distribution of particles (not anymore uniform) which, however, does produce a remarkable effect on both the variance and the tails of the acceleration pdfs. At large $S t$ values filtering effect are instead capable to explain the asymptotic behaviour of the acceleration. Present results do compare nicely (see Fig. 3) with recent state-of-the-art experimental measurements [6]. In the future it will be interesting to assess the relevance of finite density effect or of the back-reaction of the particle on the fluid itself.

\section{Acknowledgements}

We wish to thank Zellman Warhaft for sharing the experimental data. This work has been partially supported by the EU under contract HPRN-CT-200200300 and the Galileo program on particle suspensions. DNS were performed at CINECA (Italy) and IDRIS (France) under the HPC-Europa programme (RII3-CT-2003-506079). The unprocessed data of this study are freely available from http://cfd.cineca.it.

\section{References}

1. Bec J, Biferale L, Cencini M, Lanotte AS, Musacchio S, Toschi F (2006) Clusters and voids of heavy particles in turbulent flows. In this same volume.

2. Maxey MR, Riley J (1983) Phys Fluids 26:883-889

3. Chen S, Doolen GD, Kraichnan RH, She ZS (1993) Phys Fluids A 5:458-463

4. Sawford BL, Yeung PK, Borgas MS, Vedula P, La Porta A, Crawford AM, Bodenschatz E (2003) Phys Fluids 15: 3478-3489

5. La Porta A, Voth GA, Crawford AM, Alexander J, Bodenschatz E (2001) Nature 409:1017

6. Ayyalasomayajula S, Gylfason A, Collins LR, Bodenschatz E, Warhaft Z (2006) Phys Rev Lett 97:144507

7. Bec J, Biferale L, Boffetta G, Celani A, Cencini M, Lanotte A, Musacchio S, Toschi F (2006) J Fluid Mech 550:349-358

8. Cencini M, Bec J, Biferale L, Boffetta G, Celani A, Lanotte A, Musacchio S, Toschi F (2006) J Turbul 7:1-16 